\title{
Browsing and Experiencing Repositories of Spatially Oriented Historic Photographic Images
}

\author{
JONAS BRUSCHKE, Julius-Maximilians-Universität Würzburg, Germany \\ FERDINAND MAIWALD, Technische Universität Dresden, Germany \\ SANDER MÜNSTER, Technische Universität Dresden, Germany \\ FLORIAN NIEBLING, Julius-Maximilians-Universität Würzburg, Germany
}

\begin{abstract}
Many institutions archive historical images of architecture in urban areas and make them available to scholars and the general public through online platforms. Users can explore these often huge repositories by faceted browsing or keyword-based searching. Metadata that enable these kinds of investigations, however, are often incomplete, imprecise, or even wrong. Thus, retrieving images of interest can be a cumbersome task for users such as art and architectural historians trying to answer their research questions. Many of these images, often containing historic buildings and landscapes, can be oriented spatially using automatic methods such as "structure from motion" (SfM). Providing spatially and temporally oriented images of urban architecture, in combination with advanced searching and exploration techniques, offers new potential in supporting historians in their research. We are developing a 3D web environment useful to historians enabling them to search and access historic photographic images in a spatial context. Related projects use 2D maps, showing only a planar view of the current urban situation. In this paper, we present an approach to create interactive views of 4D city models, i.e., 3D spatial models that show changes over time, to provide a better understanding of the urban building situation regarding the photographer's position and surroundings. A major feature of the application is to make it possible to spatially align 3D reconstruction models to photogrammetric digitized models based on historical photographs. At the same time, this mixed methods approach is used for validation of the 3D reconstructions.
\end{abstract}

Key words:

Image repositories, 4D browser, 3D Web, Architecture.

SDH Reference:

Jonas Bruschke et al. 2018. Browsing and Experiencing Repositories of Spatially Oriented Historic

Photographic Images. SDH, 2, 2, 138-149.

DOI : $10.14434 /$ sdh.v2i2.24460

\footnotetext{
The work presented in this paper has been funded by the German Federal Ministry of Education and Research (BMBF) as part of the research project "HistStadt4D - Multimodale Zugänge zu historischen Bildrepositorien zur Unterstützung stadt- und baugeschichtlicher Forschung und Vermittlung", grant identifier 01UG1630A/B.

Author's address: Jonas Bruschke and Florian Niebling, Human-Computer Interaction, Universität Würzburg, Am Hubland, 97074 Würzburg, Germany; email: (jonas.bruschke, florian.niebling)@uni-wuerzburg.de; Ferdinand Maiwald and Sander Münster, Technische Universität Dresden, Medienzentrum, 01062 Dresden, Germany; email: (ferdinand.maiwald, sander.muenster)@tu-dresden.de.

( [2018] by the authors; licensee Studies in Digital Heritage, IU, Bloomington (IN), USA. This article is an open access article distributed under the terms and conditions of the Creative Commons Attribution License (CC BY-NC).
} 


\section{INTRODUCTION}

Historical photographs often contain highly dense information and are of great importance as sources in the humanities, in particular in the fields of Art History and Architectural History. Many institutions archive historical images of architecture in urban areas and make them available to scholars and the general public through online platforms. Many researchers rely on these often huge repositories to answer their research questions. Users can explore them by faceted browsing or keyword-based searching (Fig. 1). However, the results depend heavily on the quality of the keywords and annotations attached to the image. Keywords and other metadata are often incomplete, imprecise, vague, not standardized, or even wrong. Hence, users might obtain results that only vaguely resemble entered keywords, while more suitable content is not listed at all. Extensive digital libraries often fail to meet the expectations and requirements of scholars [Friedrichs et al. 2018]. For those scholars, it is a difficult and cumbersome task to find all the material that is potentially of interest. It is even more difficult for non-experts who want to explore old photographs and who either do not know the specific terms for which to search, or who are not familiar with the city's buildings. Many scholars relying on visual media note that online searching for images and information is "counter-productive" owing to the great amount of irrelevant data they come across or because of their own limited technical abilities [Beaudoin and Brady 2011]. The degree of search expertise certainly exerts an influence on research progress and results [Kemman et al. 2014]. Moreover, many existing tools for research platforms and applications neither meet the needs of scholars from the humanities nor take into account their behavior and skills [Alexander 2010; Warwick 2012; Dudek et al. 2015].

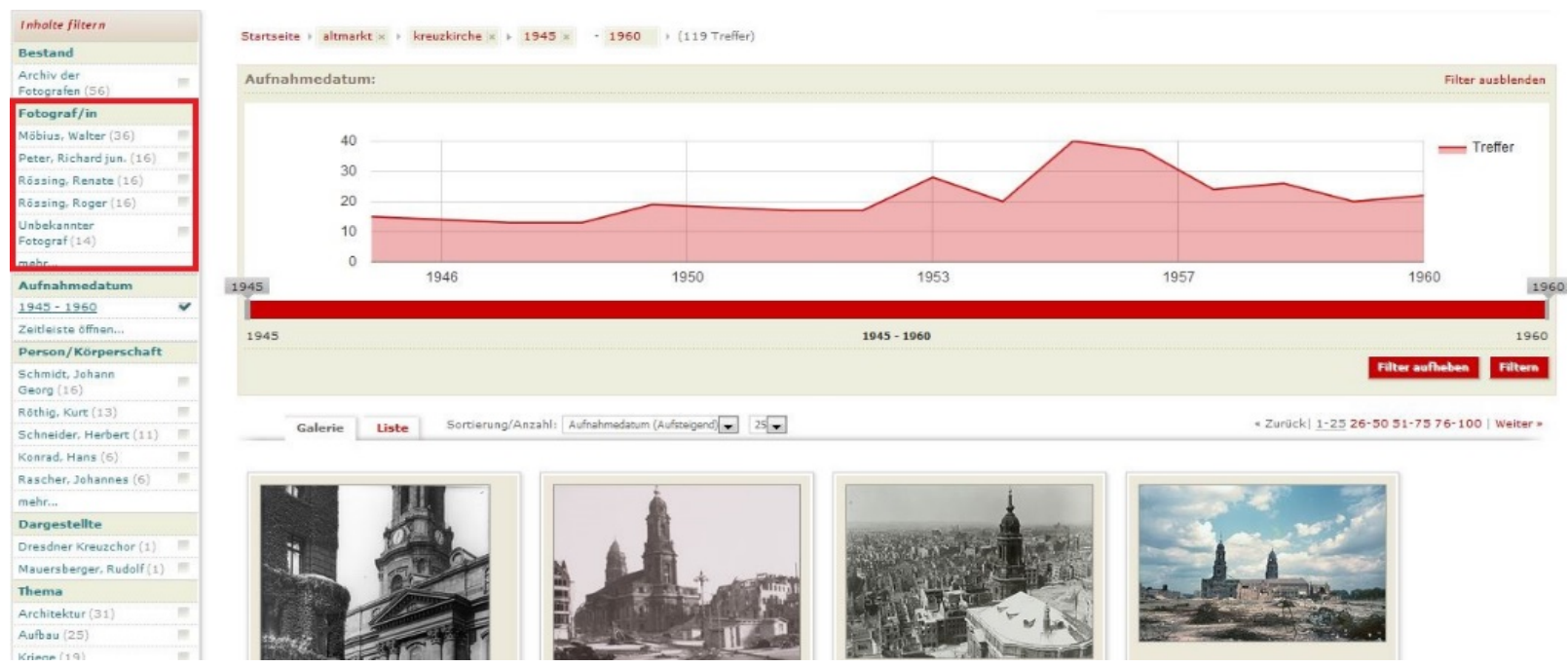

Figure 1. Possibilities of filtering the results [Sächsische Landesbibliothek - Staats - und Universitätsbibliothek Dresden 2019].

There are already attempts to improve and automatize annotation of photographic images. While automatic semantic indexing is quite evolved for textual documents [Manning et al. 2008], unlocking semantics from images is far more difficult and hence less developed. Images can be indexed by 
extracting color, texture, and shape features so that images with similar characteristics can be queried [Vassilieva 2009]. These content-based image retrieval (CBIR) methods do not generally extract semantic information that is interesting to humans. In recent years, machine learning and deep learning techniques have evolved to bridge the gap between "low-level image pixels captured by machines and high-level semantic concepts perceived by human" [Wan et al. 2014]. These techniques, however, need large training datasets and are restricted to the data they have learned. There is also some degree of probability that wrong features will be detected and associated [Llamas et al. 2016]. Processing historical photographs is even more difficult as there is less information available owing to a lack of color features.

The ARTigo project [Wieser et al. 2013] implements a crowdsourcing approach: the process of indexing images is wrapped into an online game where users can interact with images of artworks. The users' semantic footprints are processed and used to annotate these artworks. One weakness of this approach is that non-experts can also contribute to this so-called "social tagging", and, as a result, key terms that are used by professionals might be missing.

We have pursued a different approach. Many images-especially those depicting historic buildings and landscapes - can be spatially oriented using automatic methods such as "structure from motion" (SfM). Providing spatially and temporally oriented images of urban architecture, in combination with advanced searching techniques, offers new potential support for historical research. We are developing a 3D web environment that combines (historical) 3D city models and images of architecture to provide a spatial as well as temporal mode of exploration in addition to metadatabased access.

\section{RELATED WORK}

While the idea of spatially searching for images is not new, the combination of spatial images with 3D city models is not yet fully implemented. Automatic reconstruction of entire cities from images has also been already achieved, but the application to historical photographs is far more challenging and hence still in its infancy.

\section{$2.12 \mathrm{D}$ approaches}

A simple approach to explore spatially oriented images makes use of interactive 2D maps. Google Maps/Earth and Flickr are just two examples of how images can be searched by location. However, these images first have to be located by the contributing users and are usually photographs shot fairly recently. Modern handheld devices might even automatically geo-tag pictures as they are shot. But particularly with regard to historical images, the appropriate location has to be determined.

Several online platforms exist that implement interactive 2D maps and focus on historical images and where some museums and archives are contributing collections of photographs. Users can geotag these images [Historypin 2019] or can upload their own images [Varlamov and Duk 2017]. Comparison with an equivalent view today can be realized when a user re-photographs the same location [Estonian Photographic Heritage Society 2019], matching the nearest Google Street View location [City of Philadelphia 2019], or matching modern photographs by manually stitching together 
several corresponding points [Gouveia et al. 2015]. In the case of popular places with many picture entries, the image representations (or markers) are usually clustered to retain clarity.

All of these platforms utilize Google Maps, OpenStreetMap, or some such similar map service. However, 2D maps can only visualize the location and orientation of an image or rather the corresponding camera, but not the elevation or tilt. The transfer to Google Street View only works if the photograph has been shot on a street. References to historic building states are also missing, as these maps obviously show only the current state of construction.

\section{$2.23 \mathrm{D}$ approach}

Schindler and Dellaert [2012] introduced the third dimension within their approach. In a 3D environment, the user is not restricted to an orthogonal top view on a map but can also navigate at custom angles and take up the position and orientation of either the camera or the photographer. This enables a more detailed understanding of the distribution of the photographs, and a subtler access to comprehend the photographer's position. However, a 3D city model is needed to understand the relationship between building structures and the position of the camera. If images were taken above ground level, a 3D model can reveal if they were taken from rooftops or upper stories of neighboring buildings.

\subsection{D content retrieval and generation}

Since cities change over time, 3D city models have to be variable in time as well. To obtain such 4D city models, different methods are available. 3D models of the current building situation can be retrieved from city departments that hold models in different levels of detail (LOD1 to LOD3). The streaming and rendering of huge datasets of those city models in web environments have been treated in previous scholarship [Gaillard et al. 2015; Prandi et al. 2015; Nguyen et al. 2016].

Past buildings are often manually reconstructed from various sources. These sources, e.g., plans or images, can be interpreted in different ways. While new scientific findings can be obtained during the process of reconstruction [Echtenacher 2011], there is still some uncertainty about the final reconstruction in the majority of cases [Bentkowska-Kafel et al. 2012]. Projects to reconstruct single buildings are generally complex and time-consuming tasks. Accordingly, a project to reconstruct an entire city is rare and generally exceedingly difficult.

In the last fifteen years with increasing computing power, photogrammetric methods have evolved into automatic processes, i.e., "structure from motion" (SfM), capable of reconstructing whole cities from large collections of (mostly touristic) images found on the Internet [Snavely et al. 2006; Agarwal et al. 2011]. Though, they primarily considered modern photographs. Schindler and Dellaert [2010] developed an algorithm to temporally infer and sort images and to automatically detect changes in structure over time. This probabilistic method is based on SfM and can also produce 3D points and 3D geometry of buildings enhanced with time intervals. This, however, only worked with images from the 1950s and later, "due to the inability to detect corresponding SIFT features" in older images [Schindler and Dellaert 2012]. For those older images, an interactive approach was employed, whereby users could identify corresponding points and "define a building by joining a series of points." 
Other research showed that it is possible to retrieve 3D information from historical monochrome images using photogrammetry [Grün et al. 2004; Falkingham et al. 2014]. However, only a very selective dataset of images was used. General workflows for contemporary photographs that were simply applied to historical images often failed owing to several reasons. Most important among these were radiometrical differences of the digitalized images, illumination or weather changes, and object changes in the images. All these led to errors in automatic feature detection [Ali and Whitehead 2014]. So, many researchers still rely on user interaction for feature matching [Lee et al. 2011; Schindler and Dellaert 2012], while others are trying to improve recent concepts [Stylianou et al. 2015; Wahbeh and Nebiker 2017].

\section{TOWARDS A 4D IMAGE BROWSER}

To summarize: existing platforms implementing a spatial search have been directed to the general public and non-experts. Advanced features that are requested by historians are lacking. Our goal is to create a research platform that addresses the requirements of experts in the first instance, but which can also be used by non-experts.

A first, basic prototype has been developed, which can search for images spatially, temporally and by keywords. The user interface has a basic search bar into which keywords can be entered. The search results are presented in two ways (Fig. 2): on the right is a rather standard list that shows a thumbnail of each image together with the most important metadata, i.e., title, author, and date.

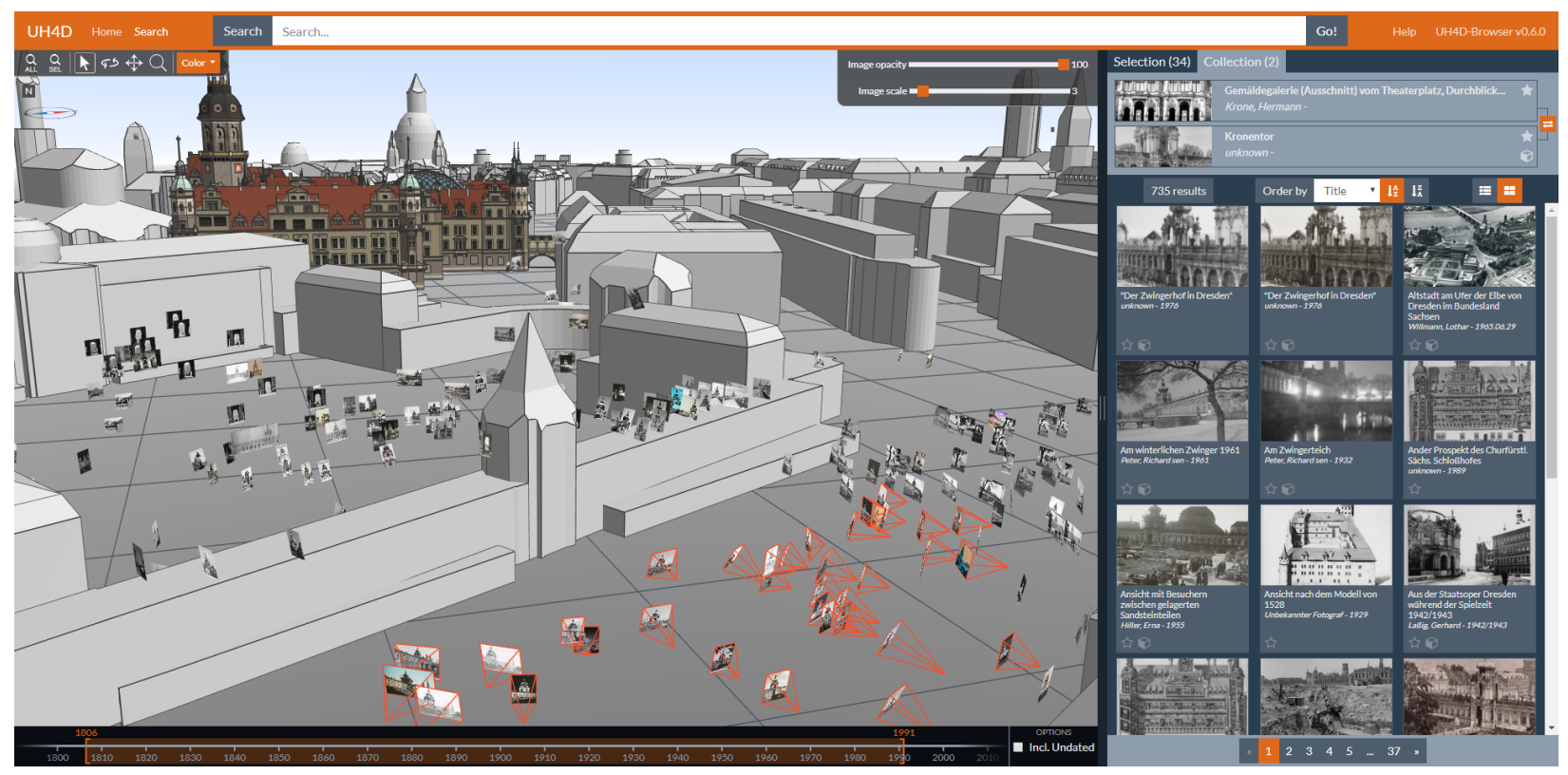

Figure 2. Prototype user interface of the application.

A small icon indicates if the image has already been spatially oriented. On the left, the 3D viewport displays the 3D city model and all oriented images. By interaction with the 3D scene, e.g., selecting buildings or defining an area, the images are filtered, so only those images that show the building or 
are within the area remain. A timeline at the bottom enables the user to narrow the results temporally. It is possible to take up the position of the camera and to toggle between the photograph and the 3D model [Bruschke et al. 2017]. On image selection, an additional view displays extended metadata information and an image viewer enabling a more detailed inspection of the image.

Designing and developing an application, which integrates a 3D environment to spatially search for orientated images, implies various challenges that range from image orientation 3D content generation problems to aspects of usability and visualization of the 3D environment and its contents.

\subsection{Automatic image orientation and 3D reconstruction}

Related work successfully applied SfM techniques to fairly old [Schindler and Dellaert 2010] or just highly selective photographs [Grün et al. 2004; Falkingham et al. 2014] (see above). While history of photography basically spans from the mid of the 19th century until today, we are primarily focusing on old, monochrome photographs and how to automatically process those across a broader, more general dataset. This task is far more difficult: there are fewer images available for processing. The majority is monochrome and does not contain any color information. Image noise emerges owing to film grain, dust particles or the digitalization process. There exists almost no information about the camera used and its orientation. Further challenges for automatic processing are radiometrical differences as well as structural changes over time across images.

We have already managed to (semi-automatically) orient historic images with the help of modern images (as a "gap-closer" between historical images). It has also been possible to generate a 3D model representing the historic state of a building from these oriented images [Maiwald et al. 2017]. Not all of the historic buildings in the images still exist, so modern photographs cannot always be applied in this process. Hence, we need to intervene in the SfM pipeline and enhance it to meet our purposes. Therefore, we plan to abandon the classical approach using SIFT features (and thus intensity-based descriptors) [Lowe 2004]. In a first approach, our workflow will use geometric features and semantic properties of quadrilaterals to improve feature detection [Maiwald et al. 2018].

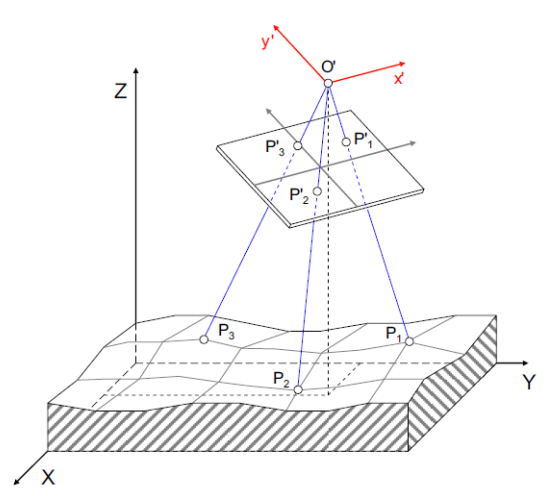

a)

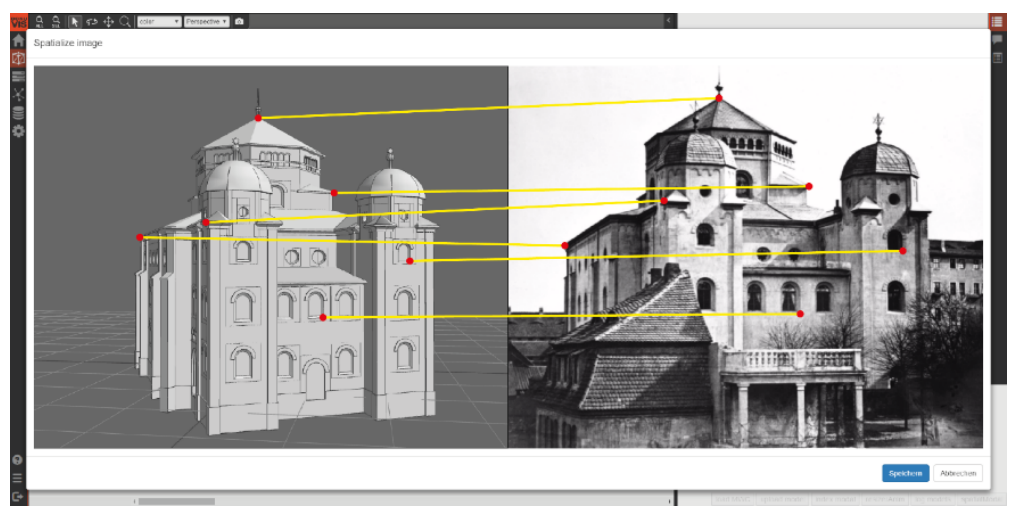

b)

Figure 3. a) Direct Linear Transformation [Luhmann 2010]; b) User interface to identify corresponding points in 3D model and photograph. 
Until a working solution for automatic image orientation is available, we have to rely on user interaction. To manually integrate images into an existing 3D scene, the user can identify corresponding points on the 3D model and in the photograph (Fig. 3). The image is then automatically oriented by applying the Direct Linear Transformation [Luhmann 2010]. However, this only works if the 3D model is detailed enough. LOD1 or LOD2 models are usually too rough and abstract to properly match corresponding points resulting in unusable image orientation. As a fallback, it is also possible to overlay the 3D scene with the image and to manually align the image with the view.

\subsection{Usability concerns}

Usability is the degree to which a product is easy to use. It is an important aspect that should not be neglected when designing applications. Applications designed without reference to user requirements remain unused or unappreciated [Warwick 2012]. The proposed application needs to approach the gap between professional use by experts and a rather simple exploration by nonexperts. It is also important to adopt established features and techniques to which users are already accustomed. So, keyword-based and faceted search are not replaced but extended by means of spatial and temporal exploration.

User studies also showed that people, who are not used to 3D environments, "can get into navigation trouble very quickly (...) and once in trouble, have a hard time getting back to a known, comfortable viewing state" [Fitzmaurice et al. 2008]. This can result in frustration and rejection of the application. A possible fallback to a top view with simple panning and zooming might solve this problem. Preferably, advanced navigation tools that take into account experienced users as well as those new to 3D should be adopted to ensure usability for everyone [Fitzmaurice et al. 2008].

\subsection{Visualization}

\subsubsection{D content}

The visualization itself is also part of the usability and the user experience of the application. The architecture, 3D content, and information offered need to be properly communicated to the user to prevent misconceptions. This includes the clear differentiation of $3 \mathrm{D}$ models representing the modern and historic building states. 3D models of past structures might be incomplete or noisy owing to a lack of information behind either the manual reconstruction or the SfM algorithm employed. These objects need to be integrated and indicated properly, so that the user is able to distinguish between historic and current states of the city. Appropriate visualization methods still need to be evaluated when first reconstruction results are available. Especially at places where no buildings could be reconstructed, orientation within the historic city can be supported by historic maps on ground level.

\subsubsection{Images}

In 3D environments, images are usually displayed as textured planes, sometimes enhanced by a frustum [Snavely et al. 2006] to indicate the orientation and angle of view (cf. Fig. 2). At places with a high density of images, this can lead to intersection as well as to (partial) occlusion. Orientation and 
exact position of images are generally only of interest when viewing at close range. Looking from farther away, the images can have an offset to their position, always face the user's camera, and be automatically arranged to prevent occlusion [Chagnaud et al. 2016]. Especially when viewing a 3D city model from farther away, many images would be hidden by buildings and thus hard to spot. The visualization of the images needs to dynamically adapt depending on the user's point of view. At close range, the exact position and orientation are of interest (i.e., frustum or pyramid of vision). When looking from farther away, another presentation mode is more suitable, e.g., images floating above buildings as billboards. A smooth, interpolated transition between the different presentation modes at certain distances is reasonable. For hotspots of images, additional methods might be necessary, e.g., clustering, weighting, or filtering images by discriminative features [van Leuken et al. 2009].

\subsubsection{Uncertainty and reliability}

The 3D city model representing the current building situation will be most likely quite complete and accurate. If buildings are presented in a low level of detail, it is not the result of a lack of information, but more for reasons of abstraction. 3D models representing past city structures are reconstructed either manually or by photogrammetric methods. Either way, it is not guaranteed that the reconstruction will be accurate or even correct. The resulting uncertainty needs to be indicated, and it is necessary to communicate the fact that these reconstructions are just hypotheses about the historic building state. Otherwise, the user would assume that visualized structures are factual, resulting in miscommunication.

In the field of cultural heritage and digital, interpretative, and hypothetical 3D reconstructions, this matter is of high significance [Bentkowska-Kafel et al. 2012]. There are many approaches to indexing and visualizing uncertainty or reliability. The methods employed range from transparency, wireframe rendering [Lengyel and Toulouse 2011], different level of details to color-coding the elements [Apollonio 2016]. A combination of multiple methods is also possible [Kensek et al. 2004]. The amount of uncertainty depends on the quality of sources that led to the reconstruction and is defined by those who carried out the reconstruction.

By applying photogrammetric methods, we strive to automatize the process of image orientation and 3D reconstruction. The output of this process greatly depends on the quality of the images. In this respect, an evaluation of this output according to its accuracy and deviation is needed. A proper (automated) validation process is still to be developed. For that, some accurate model is required as reference to check against. Additionally, the photogrammetric methods applied can provide probabilistic values to their results. Finally, both images and 3D models have to be indexed and visualized according to their determined probabilistic reliability.

\section{CONCLUSION AND FUTURE WORK}

Most existing solutions rely on 2D map services. They are easy to integrate into webpages, they provide map material and satellite imagery of the entire earth, and their APIs offer extensive geospatial functionalities. However, the maps provided show only the current building state. The extension using 3D city models is considered to be beneficial in regard to better identification of the 
region of interest, a better understanding of the urban building situation, as well as the photographer's point of view. Because the historic building condition can differ significantly from the current state, those 3D city models need to be temporal, or 4D. While contemporary parts of the 3D model can often be retrieved from city departments, photogrammetric methods need to be evaluated and further developed to automatically generate 3D models of historic buildings. It is, however, possible that only parts of a city can be reconstructed owing to a lack of the data that would be required. The user must be clearly informed about which 3D models represent the contemporary building state and which the historic. This also applies to the uncertainty and inaccuracy of reconstructed historic building and oriented images. The validation of 3D models and image orientation according to their accuracy and reliability still is a major challenge.

The research environment for art and architectural historians introduced in this work needs to be able to visualize and communicate different kinds of information without confusing the user. Although development happens in close collaboration with historians, user tests on a broader audience still need to be performed. Tools to take measurements and to better analyze distribution of the images as well as composition of photographs will be developed to support historians in their research objectives.

\section{REFERENCES}

Sameer Agarwal, Yasutaka Furukawa, Noah Snavely, Ian Simon, Brian Curless, Steven M. Seitz, and Richard Szeliski. 2011. Building Rome in a Day. Communications of the ACM54, 10 (2011), 105-112. DOI:http://dx.doi.org/10.1145/2001269.2001293

Maxwell Alexander. 2010. Digital archives and history research: feedback from an end-user. Library Review59, 1, 24-39. DOI:http://dx.doi.org/doi:10.1108/00242531011014664

Heider K. Ali and Anthony Whitehead. 2014. Feature Matching for Aligning Historical and Modern Images. International Journal of Computers and Their Applications 21, 3, 188-201.

Fabrizio I. Apollonio. 2016. Classification Schemes for Visualization of Uncertainty in Digital Hypothetical Reconstruction. In Sander Münster, Mieke Pfarr-Harfst, Piotr Kuroczyński, and Marinos Ioannides, eds. 3D Research Challenges in Cultural Heritage II: How to Manage Data and Knowledge Related to Interpretative Digital 3D Reconstructions of Cultural Heritage. Cham: Springer International Publishing, 173-197. DOI: http://dx.doi.org/10.1007/978-3-319-47647-6_9

Joan E. Beaudoin and Jessica Evans Brady. 2011. Finding Visual Information: A Study of Image Resources Used by Archaeologists, Architects, Art Historians, and Artists. Art Documentation: Journal of the Art Libraries Society of North America 30, 2 (2011), 24-36.

Anna Bentkowska-Kafel, Hugh Denard, and Drew Baker. 2012. Paradata and Transparency in Virtual Heritage. Burlington: Ashgate.

Jonas Bruschke, Florian Niebling, Ferdinand Maiwald, Kristina Friedrichs, Markus Wacker, and Marc Erich Latoschik. 2017. Towards Browsing Repositories of Spatially Oriented Historic Photographic Images in 3D Web Environments. In Proceedings of the 22nd International Conference on 3D Web Technology (Web3D '17). Brisbane, Australia, ACM. DOI:http://dx.doi.org/10.1145/3055624.3075947

Clément Chagnaud, John Samuel, Sylvie Servigne, and Gilles Gesquière. 2016. Visualization of Documented 3D Cities. In Eurographics Workshop on Urban Data Modelling and Visualisation. 
Liège, Belgium, The Eurographics Association. DOI: http://dx.doi.org/10.2312/udmv.20161425 Weiqin Chen and Thomas Nottveit. 2010. Digital Map Application for Historical Photos. In Gobinda Chowdhury, Chris Koo, and Jane Hunter, eds. The Role of Digital Libraries in a Time of Global Change: 12th International Conference on Asia-Pacific Digital Libraries, ICADL 2010, Gold Coast, Australia, June 21-25, 2010. Proceedings. Berlin, Heidelberg: Springer, 158-167.

DOI:http://dx.doi.org/10.1007/978-3-642-13654-2_20

City of Philadelphia. 2019. The Philadelphia City Archive. Retrieved February 25, 2019 from https://www.phillyhistory.org

Sächsische Landesbibliothek - Staats - und Universitätsbibliothek Dresden. 2019. Deutsche Fotothek. Retrieved February 25, 2019 from http://www.deutschefotothek.de

Iwona Dudek, Jean-Yves Blaise, Livio De Luca, Laurent Bergerot, and Noémie Renaudin. 2015. How was this done? An attempt at formalising and memorising a digital asset's making-of. In Proceedings of the 2nd International Congress on Digital Heritage Vol. 2. IEEE, 343-346. DOI:http://dx.doi.org/10.1109/DigitalHeritage.2015.7419519

Götz Echtenacher. 2011. Wissenschaftliche Erkenntnisse durch manuelles Konstruieren von 3DModellen. In Katja Heine, Klaus Rheidt, Frank Henze, and Alexandra Riedel, eds. Von Handaufmaß bis High Tech III. Darmstadt/Mainz: Verlag Philipp von Zabern, 49-57.

Estonian Photographic Heritage Society. 2019. Ajapaik, a crowdsourcing platform for geotagging historic images. Retrieved February 25, 2019 from https://ajapaik.ee

Peter L. Falkingham, Karl T. Bates, and James O. Farlow. 2014. Historical Photogrammetry: Bird's Paluxy River Dinosaur Chase Sequence Digitally Reconstructed as It Was prior to Excavation 70 Years Ago. PLOS ONE, 9, 4 (2014), e93247. DOI:http://dx.doi.org/10.1371/journal.pone.0093247

George Fitzmaurice, Justin Matejka, Igor Mordatch, Azam Khan, and Gordon Kurtenbach. 2008. Safe 3D Navigation. In Proceedings of the 2008 Symposium on Interactive 3D Graphics and Games. Redwood City, California, ACM, 7-15. DOI:http://dx.doi.org/10.1145/1342250.1342252

Kristina Friedrichs, Sander Münster, Cindy Kröber, and Jonas Bruschke. 2018. Creating Suitable Tools for Art and Architectural Research with Historic Media Repositories. In Sander Münster, Kristina Friedrichs, Florian Niebling, and Agnieszka Seidel-Grzesińska, eds. Digital Research and Education in Architectural Heritage. UHDL 2017, DECH 2017. Cham: Springer International Publishing, 117-138. DOI:http://dx.doi.org/10.1007/978-3-319-76992-9_8

Jérémy Gaillard, Alexandre Vienne, Rémi Baume, Frédéric Pedrinis, Adrien Peytavie, and Gilles Gesquière. 2015. Urban Data Visualisation in a web browser. In Proceedings of the 20th International Conference on 3D Web Technology (Web3D '15). Heraklion, Crete, Greece, ACM, 8188. DOI:http://dx.doi.org/10.1145/2775292.2775302

João Gouveia, Fernando Branco, Armanda Rodrigues, and Nuno Correia. 2015. Travelling Through Space and Time in Lisbon's Religious Buildings. In Proceedings of the 2nd International Congress on Digital Heritage Vol. 2. IEEE, 407-408. DOI:https://doi.org/10.1109/DigitalHeritage.2015.7413916

Armin Grün, Fabio Remondino, and Li Zhang. 2004. Photogrammetric Reconstruction of the Great Buddha of Bamiyan, Afghanistan. The Photogrammetric Record, 19, 107 (2004), 177-199.

DOI:http://dx.doi.org/10.1111/j.0031-868X.2004.00278.x

Historypin. 2019. Connecting communities with local history. Retrieved February 26, 2019 from https://www.historypin.org 
Max Kemman, Martijn Kleppe, and Stef Scagliola. 2014. Just Google It - Digital Research Practices of Humanities Scholars. In Clare Mills, Michael Pidd, and Esther Ward, eds. Proceedings of the Digital Humanities Congress 2012. Studies in the Digital Humanities. Sheffield: HRI Online Publications.

Karen M. Kensek, Lynn Swartz Dodd, and Nicholas Cipolla. 2004. Fantastic reconstructions or reconstructions of the fantastic? Tracking and presenting ambiguity, alternatives, and documentation in virtual worlds. Automation in Construction, 13, 175-186.

Kun-Ting Lee, Sheng-Jie Luo, and Bing-Yu Chen. 2011. Rephotography Using Image Collections. Computer Graphics Forum, 30, 7 (2011), 1895-1901. DOI:http://dx.doi.org/10.1111/j.14678659.2011.02042.X

Dominik Lengyel and Catherine Toulouse. 2011. Darstellung von unscharfem Wissen in der Rekonstruktion historischer Bauten. In Katja Heine, Klaus Rheidt, Frank Henze, and Alexandra Riedel, eds. Von Handaufmaß bis High Tech III. Darmstadt/Mainz: Verlag Philipp von Zabern, 182-187.

Jose Llamas, Pedro M. Lerones, Eduardo Zalama, and Jaime Gómez-García-Bermejo. 2016. Applying Deep Learning Techniques to Cultural Heritage Images Within the INCEPTION Project. In Marinos Ioannides, Eleanor Fink, Antonia Moropoulou, Monika Hagedorn-Saupe, Antonella Fresa, Gunnar Liest $\varnothing$ l, Vlatka Rajcic, and Pierre Grussenmeyer, eds. Digital Heritage. Progress in Cultural Heritage: Documentation, Preservation, and Protection: 6th International Conference, EuroMed 2016, Nicosia, Cyprus, October 31 - November 5, 2016, Proceedings, Part II. Cham: Springer International Publishing, 25-32. DOI:http://dx.doi.org/10.1007/978-3-319-48974-2_4

David G. Lowe. 2004. Distinctive Image Features from Scale-Invariant Keypoints. International Journal of Computer Vision 60, 2 (2004), 91-110.

DOI:http://dx.doi.org/10.1023/b:visi.0000029664.99615.94

Thomas Luhmann. 2010. Nahbereichsphotogrammetrie: Grundlagen, Methoden und Anwendung. Berlin: Wichmann.

Ferdinand Maiwald, Danilo Schneider, and Frank Henze. 2018. Bestimmung der Korrespondenz zwischen historischen Gebäudeaufnahmen basierend auf der Zuordnung geometrischer Merkmale. In Publikationen der Deutschen Gesellschaft für Photogrammetrie, Fernerkundung und Geoinformation e.V., 38. Wissenschaftlich-Technische Jahrestagung der DGPF, 07.-09. März 2018 in München.

Ferdinand Maiwald, Theresa Vietze, Danilo Schneider, Frank Henze, Sander Münster, and Florian Niebling. 2017. Photogrammetric Analysis of Historical Image Repositories for Virtual Reconstruction in the Field of Digital Humanities. Int. Arch. Photogramm. Remote Sens. Spatial Inf. Sci. XLII-2/W3 (2017), 447-452. DOI:http://dx.doi.org/10.5194/isprs-archives-XLII-2-W3-4472017

Christopher D. Manning, Prabhakar Raghavan, and Hinrich Schütze. 2008. Introduction to Information Retrieval. Cambridge: Cambridge University Press.

DOI:http://dx.doi.org/10.1017/CB09780511809071

Quoc-Dinh Nguyen, Mathieu Bredif, Didier Richard, and Nicolas Paparoditis. 2016. Progressive streaming and massive rendering of 3D city models on web-based virtual globe. In Proceedings of the 24th ACM SIGSPATIAL International Conference on Advances in Geographic Information Systems. Burlingame, California, ACM. DOI:http://dx.doi.org/10.1145/2996913.2997008 
Federico Prandi, Federico Devigili, Marco Soave, Umberto Di Staso, and Raffaele De Amicis. 2015. 3D web visualization of huge CityGML models. Int. Arch. Photogramm. Remote Sens. Spatial Inf. Sci. XL-3/W3 (2015), 601-605. DOI:http://dx.doi.org/10.5194/isprsarchives-XL-3-W3-601-2015

Grant Schindler and Frank Dellaert. 2010. Probabilistic Temporal Inference on Reconstructed 3D Scenes. In 2010 IEEE Conference on Computer Vision and Pattern Recognition (CVPR). IEEE, 1410-1417. DOI:http://dx.doi.org/10.1109/CVPR.2010.5539803

Grant Schindler and Frank Dellaert. 2012. 4D Cities: Analyzing, Visualizing, and Interacting with Historical Urban Photo Collections. Journal of Multimedia 7, 2(2012), 124-131. URI:http://hdl.handle.net/1853/48719

Noah Snavely, Steven M. Seitz, and Richard Szeliski. 2006. Photo Tourism: Exploring Photo Collections in 3D. ACM Transactions on Graphics (SIGGRAPH Proceedings)25, 3 (2006), 835-846. DOI:http://dx.doi.org/10.1145/1141911.1141964

Abby Stylianou, Austin Abrams, and Robert Pless. 2015. Characterizing Feature Matching Performance over Long Time Periods. In 2015 IEEE Winter Conference on Applications of Computer Vision. Waikoloa, HI, USA, IEEE, 892-898. DOI:http://dx.doi.org/10.1109/WACV.2015.123

Reinier H. van Leuken, Lluis Garcia, Ximena Olivares, and Roelof van Zwol. 2009. Visual Diversification of Image Search Results. In Proceedings of the 18th International Conference on World Wide Web. Madrid, Spain, ACM, 341-350. DOI:http://dx.doi.org/10.1145/1526709.1526756

Ilya Varlamov and Alex Duk. 2017. PastVu - project to collect vintage images. A look at the history of humanity habitat. Retrieved February 25, 2019 from https://pastvu.com

N. S. Vassilieva. 2009. Content-based image retrieval methods. Programming and Computer Software 35, 3 (2009), 158-180. DOI:http://dx.doi.org/10.1134/s0361768809030049

Wissam Wahbeh and Stephan Nebiker. 2017. Three Dimensional Reconstruction Workflows for Lost Cultural Heritage Monuments Exploiting Public Domain and Professional Photogrammetric Imagery. ISPRS Ann. Photogramm. Remote Sens. Spatial Inf. Sci. IV-2/W2 (2017), 319-325. DOI:http://dx.doi.org/10.5194/isprs-annals-IV-2-W2-319-2017

Ji Wan, Dayong Wang, Steven Chu Hong Hoi, Pengcheng Wu, Jianke Zhu, Yongdong Zhang, and Jintao Li. 2014. Deep Learning for Content-Based Image Retrieval: A Comprehensive Study. In Proceedings of the 22nd ACM international conference on Multimedia. Orlando, Florida, USA, ACM, 157-166. DOI:http://dx.doi.org/10.1145/2647868.2654948

Claire Warwick. 2012. Studying users in digital humanities. In Claire Warwick, Melissa Terras, and Nyhan Julianne, eds. Digital Humanities in Practice. London: Facet Publishing, 1-21.

Christoph Wieser, François Bry, Alexandre Bérard, and Richard Lagrange. 2013. ARTigo: Building an Artwork Search Engine with Games and Higher-Order Latent Semantic Analysis. In Proceedings of Disco 2013, Workshop on Human Computation and Machine Learning in Games. Palm Springs, CA, USA.

Received September 2018; revised October 2018; accepted December 2018. 